\title{
EDITORIAL
}

\section{Advancing research and practice in perinatal mental health}

\author{
Barbara Figueiredo, ${ }^{1}$ iD Erika S.V. Abuchaim ${ }^{2}$ iD \\ ${ }^{1}$ Portuguese Speaking Group, International Marcé Society for Perinatal Mental Health, Centro de Investigação em Psicologia, Escola de \\ Psicologia, Universidade do Minho, Portugal. ${ }^{2}$ Portuguese Speaking Group, International Marcé Society for Perinatal Mental Health, Escola \\ Paulista de Enfermagem, Universidade Federal de São Paulo (UNIFESP), São Paulo, SP, Brazil.
}

The perinatal period is characterized by multiple simultaneous changes at the biological, psychological, and social levels, affecting not only the mother, but also the father and infant. While these changes typically improve the necessary conditions and skills to safeguard pregnancy and parenting, because they occur in such a short period of time, they can also give rise to psychological problems or disorders. There is a relatively high prevalence of mental health problems in the perinatal period when compared to other moments of the life cycle, with immediate and long-term adverse consequences, particularly for fetal health and child development.

The perinatal period is, therefore, a critical time for clinical interventions focused on promoting, preventing, or treating mental health problems in parents or infants. Expanding scientific research in perinatal mental health is key to improving the effectiveness of these interventions. In 2020, the Portuguese Speaking Group of the International Marcé Society for Perinatal Mental proposed to the editors of the Brazilian Journal of Psychiatry a call for papers, which resulted in three articles included in the current issue.

These articles attest to the expansion of perinatal mental health research across different countries, allowing the replication and validation of previous findings in new contexts and cultures. Faisal-Cury et al. ${ }^{1}$ explore a much important but often neglected theme - the decline of sexual life in the postpartum period, particularly among women with postpartum depression. The same is true for the study of Aydogan et al., ${ }^{2}$ who studied a group of pregnant women with panic disorder, showing alleviation of symptoms during the postpartum period. Finally, the study of Uguz \& $\mathrm{Ak}^{3}$ presents data on the effectiveness of cognitive-behavioral therapy in women with generalized anxiety disorder during pregnancy. Collectively, these articles reflect the increasing understanding of the nature, clinical course, and correlates of perinatal mental health problems, and should encourage researchers in the field to expand their own conceptual framework and research methods.

\section{Disclosure}

The authors report no conflicts of interest.

\section{References}

1 Faisal-Cury A, Tabb K, Matijasevich A. Partner relationship quality predicts later postpartum depression independently of the chronicity of depressive symptoms. Braz J Psychiatry. 2021;43:12-21.

2 Aydogan S, Uguz F, Yakut E, Bayman MG, Gezginc K. The course and clinical correlates of panic disorder during the postpartum period: a naturalistic observational study. Braz J Psychiatry. 2021;43:6-11.

3 Uguz F, Ak M. Cognitive-behavioral therapy in pregnant women with generalized anxiety disorder: a retrospective cohort study on therapeutic efficacy, gestational age and birth weight. Braz J Psychiatry. 2021;43:61-64.
Correspondence: Barbara Figueiredo, Escola de Psicologia, Universidade do Minho, Campus de Gualtar, 4710-057, Braga, Portugal.

E-mail: bbfi@psi.uminho.pt

Submitted Jan 08 2021, accepted Jan 082021.
How to cite this article: Figueiredo B, Abuchaim ESV. Advancing research and practice in perinatal mental health. Braz $\mathrm{J}$ Psychiatry. 2021;43:1. http://dx.doi.org/10.1590/1516-4446-2021-0026 